\title{
Chemical composition and FT-IR spectra of sugar palm (Arenga pinnata) fibers obtained from different heights
}

\begin{abstract}
This paper deals with the study on the effect of biological degradation to chemical composition and Fourier transform infrared (FT-IR) of sugar palm (Arenga pinnata) fibers obtained from different heights $(1,3,5,7,9,11,13$, and $15 \mathrm{~m})$ of the sugar palm tree. It was observed that degradation that took place at the bottom part of the sugar palm trunk affected the chemical composition of its fiber. It was noticed that cellulose, hemicelluloses, and lignin contents increased with the increase of the tree height. A good correlation between chemical composition and mechanical properties of sugar palm fiber was found where cellulose, lignin, and hemicelluloses show a significant contribution to the increase in tensile strength, modulus, and elongation at break of the fiber, respectively. For the fibers obtained from higher height, it was observed that cellulose and hemicelluloses contents slightly decreased. High ash content for fibers obtained from $1 \mathrm{~m}$ height resulted in low moisture content and significant increase in its thermal stability. In FT-IR study, it was determined that the relative absorbance in sugar palm fibers was no different in all fibers.
\end{abstract}

Keyword: Sugar palm fiber; Arenga pinnata; Chemical composition; Biological degradations 